\title{
Las altas de los/as socios/as en las sociedades cooperativas
}

Membership in cooperative societies

Eba Gaminde Egia1

Universidad de Deusto (España)

Sumario: I. Introducción. II. El principio de asociación voluntaria y abierta. II.1. Formulación del principio. II.2. Regulación normativa. II.3. Mención estatutaria. III. Las aportaciones. IV. Conclusión. V. Bibliografía.

Summary: I. Introduction. II. The principle of voluntary and free membership. II.1. Formulation of the principle. II.2. Regulation in legal norms. II.3. Mention in by-laws. III. The contributions. IV. Conclusions. V. Bibliography.

Resumen: En el presente trabajo analizaremos la plasmación práctica de uno de los principios cooperativos, "adhesión voluntaria y libre», refiriéndonos al alta de los y las socios y socias en las sociedades cooperativas. Para ello, explicaremos en primer lugar el significado de ese principio, para posteriormente traer a colación su regulación normativa, haciendo especial hincapié en aquellos aspectos en los que difieren nuestras leyes autonómicas, y finalizando con una breve referencia al aspecto económico y los diferentes modos de realizar las respectivas aportaciones y sus consecuencias.

Palabras clave: adhesión libre y voluntaria, requisitos del socio, procedimiento de admisión, aportación obligatoria.

Abstract: In this work we will analyze the practical application of one of the cooperative principles, "voluntary and free membership», referring to the entering of members in cooperative societies. We will first explain the meaning of this principle, and then bring up its normative regulation, with special emphasis on those aspects in which our autonomic laws differ, and ending with a brief reference to the economic aspect and the different ways to make contributions and their consequences.

Keywords: voluntary and free membership, requirements for membership, admission procedure, compulsory contribution.

1 Profesora de Derecho Mercantil en la Universidad de Deusto. Correo electrónico: eba.gaminde@deusto.es 


\section{Introducción}

En general, la entrada de un socio/a de una Sociedad Cooperativa se convierte en un proceso bastante más complicado que la entrada de un accionista en una sociedad mercantil. La simple compra de acciones convierte a cualquier persona en accionista de la sociedad anónima, mientras que la entrada en una sociedad cooperativa resulta bastante más compleja, ya que, para comenzar, el socio/a ha de ser aceptado/a, con carácter personal, por el Consejo Rector y, en su caso, por la Asamblea, como veremos.

En cualquier caso, para ubicar nuestra presentación, hemos de recordar, brevemente y con gran simplificación, el marco en el que nos desenvolvemos, mencionando que las cooperativas están compuestas de personas que, además, aportan capital, mientras que, por el contrario, las sociedades anónimas están compuestas de capitales detrás de los cuales están las personas, físicas o jurídicas, con las que la sociedad raramente se relaciona a nivel individual como tales personas. Este tratamiento individualizado de las cooperativas a sus socios y socias genera una problemática específica que se hace más patente en las entradas y salidas de los socios, aunque también se manifiesta en otras áreas de la actividad cooperativa.

Centrándonos en la entrada de un socio/a de una cooperativa, ésta puede ser analizada desde diferentes perspectivas. Desde el punto de vista legal, se ha de hacer referencia al principio básico cooperativo que da cobertura a esa entrada, el llamado principio de «adhesión voluntaria y libre» o de «puertas abiertas», que, como veremos, impide cualquier discriminación a la hora de aceptar o denegar la admisión de un socio/a. Otro aspecto a considerar lo constituye el mecanismo contable que se debe poner en marcha cuando entra un socio/a, que se reduce a un aumento del propio y del activo exactamente igual al valor de las entradas. Por último, quedan los aspectos económicos y financieros que plantean tanto las entradas como las salidas. Por una parte, el cálculo de las aportaciones que han de realizar los socios/as en caso de entrada, y, por otra, cómo se realizan o deben realizarse estas aportaciones, ya que la cooperativa utiliza diversas fórmulas para ello.

En la presente ponencia, sin embargo, nos centraremos en dos de esas cuestiones: QUIÉN puede darse de alta en la cooperativa y CÓMO ha de hacerlo, es decir, el aspecto legal y el económico, analizando, en primer lugar, el principio cooperativo de «asociación voluntaria y abierta», su regulación normativa y su posterior desarrollo estatutario, para después referirnos brevemente al aspecto económico, 
mostrando los diferentes modos de realizar las respectivas aportaciones y sus consecuencias.

\section{El principio de asociación voluntaria y abierta}

\section{II.1. Formulación del principio}

Como bien sabemos, la organización y el funcionamiento de las cooperativas se rigen por determinados principios o normas que ponen en práctica elevados valores espirituales y se hallan consagrados universalmente. Son los denominados "principios de Rochdale», así conocidos por haber sido aplicados por primera vez de forma sistemática por la cooperativa de los «Probos pioneros de Rochdale» en 1844, sobre principios ya esbozados en experiencias precedentes².

Entre ellos se encuentra el principio de LIBRE ADHESIÓN, fundamental en toda la concepción cooperativista.

En su formulación originaria, los estatutos de Rochdale se refirieron a este principio mencionando la «libre adhesión y dimisión», para explicar de forma concreta el procedimiento de entrada y salida de los socios/as en la sociedad, con un sistema de principios simples, claros y contundentes, ya que los Pioneros de Rochdale nunca se propusieron dictar normas para todo el movimiento cooperativo, entonces incipiente, sino para orientar y consolidar su propia organización.

Más concretamente, en relación con la cuestión que aquí nos ocupa, la entrada de los y las socios/as en la cooperativa, los apartados 13 y 14 decían así3:

2 La cooperativa de Rochdale ha sido tradicionalmente reconocida como la iniciadora del movimiento cooperativo moderno, identificando a los pioneros de Rochdale como «realizadores del cooperativismo» en muchos manuales, artículos y estudios monográficos. Entre otros, Möller, E. (1986, p. 23); KAPLAN De Drimer, A. y Drimer B. (1981, p. 227); MLAdenATZ, G. (1969, p. 68); URiBe GarzÓn, C. (2001, p. 68).

No obstante, también hay opiniones en otro sentido. Carrasco (1991, p. 2), por ejemplo, puntualiza que «habría que aclarar que no fue la de los Pioneros de Rochdale la primera experiencia asociativa habida en la historia y que su carácter pionero se refiere más que a la experiencia en sí, a la elaboración por parte de sus promotores de unos estatutos propios». En la misma línea MiRAnda (2012, p. 140) señala que "el cooperativismo no nace en el año 1844: es el resultado de un proceso multisecular que se inicia en la cooperación primaria».

3 «Los estatutos de la cooperativa de Rochdale. Gestión participativa» http://www.gestionparticipativa.coop/portal/index.php?option=com_content\&view=article\&id=254:losestatutos-de-la-cooperativa-de-rochdale\&catid=37:getaways\&ltemid=364 última consulta 30-05-2017. 


\section{Adhesión Libre.}

13. Cualquier persona que desee llegar a ser miembro de la sociedad será propuesta y apoyada por dos miembros en una reunión de los directivos y de los directores; si la proposición se aprueba por una mayoría de los que estén presentes, la persona será elegible en la siguiente reunión semanal; cada candidato pagará la mitad de un chelín como cuota de admisión; una vez admitido como miembro, las participaciones podrán pagarse por medio de entregas parciales de tres penique por semana por cada participación.

Cualquier miembro que descuide la entrega de estos pagos parciales durante tres meses será castigado con una multa de seis peniques, excepto en el caso de enfermedad o de paro.

Cualquier miembro que descuide la entrega de estos pagos parciales durante seis meses será excluido; su participación o sus participaciones se venderán, y después de pagados todos los cargos necesarios, el resto se entregará a dicho miembro excluido. Ningún miembro podrá tener más de cincuenta participaciones.

14. Si los dirigentes y los miembros de la sociedad encontraran que la conducta de alguno de sus miembros es perjudicial a los intereses de la sociedad, el presidente será, en virtud de esta disposición, quien deba amonestarle, y si un miembro, después de dicha amonestación, continuara en la misma línea de conducta perjudicial le avisará de su exclusión de la sociedad en el plazo de un mes a la expiración de este plazo el miembro que ha causado perjuicios a la sociedad será excluido sin otra formalidad.

De esta forma, se describía un sencillo procedimiento para la admisión por la Sociedad de nuevos asociados/as, estableciendo que deben ser propuestos y apoyados por dos socios, fijando el pago de un moderado derecho de ingreso (mitad de un chelín) y posteriores pagos en cuotas (3 peniques/semana por participación), así como la sanción en caso de impago (multa o incluso expulsión, si el retraso se prolongaba 6 meses).

Sin embargo, las normas de Rochdale estaban adaptadas a las condiciones socioeconómicas de su época y reflejaron las ideas entonces dominantes entre sus iniciadores. Era necesario extractar los principios rochdalianos, estudiar su aplicación en épocas posteriores y adoptar una formulación genérica de los mismos. Esa labor fue emprendida por la Alianza Cooperativa Internacional (ACI), que aprobó en su Congreso de París de 1937 una primera formulación universal de los principios cooperativos, posteriormente revisada y completada en sus congresos de Viena en 1966 y de Manchester en 1995.

Si bien con anterioridad se emplearon términos como «membresía», "adhesión», en su formulación de 1995 el principio se denominó de "Asociación voluntaria y abierta» estableciendo que: 
Las cooperativas son organizaciones voluntarias, abiertas a todas las personas capaces de utilizar sus servicios y dispuestas a aceptar las responsabilidades de asociarse, sin discriminaciones raciales, políticas, religiosas, sociales o de género.

Por lo tanto,

1. La propia denominación del principio abarca dos normas diferentes pero complementarias: asociación voluntaria y abierta.

- En primer lugar se trata de una asociación «voluntaria», es decir, la persona ha de elegir voluntariamente asumir un compromiso con determinada cooperativa. El ingreso de los asociados/as debe depender de su voluntad, sin que exista imposición legal o de hecho que obligue a cualquier persona a asociarse o a permanecer dentro de ella. En ese sentido, las cooperativas tienen la especial responsabilidad de asegurar que todos los asociados/as estén plenamente comprometidos y apoyen a su cooperativa de forma voluntaria.

- Además, la asociación "abierta» indica que el ingreso a las cooperativas se halla al alcance de quien desee incorporarse a ellas; de ahí la expresión "puertas abiertas» que también se aplica habitualmente. Las condiciones para el ingreso de los asociados/as han de constar en el estatuto de las cooperativas, debiendo ser mínimas y de carácter general. No deben exigirse derechos de ingreso gravosos ni otras condiciones que resulten restrictivas o discriminatorias.

2. Por otra parte, el principio especifica dos condiciones ineludibles que deberán cumplir las personas que deseen ingresar como asociados/as en la cooperativa:

- «Personas capaces de utilizar sus servicios», reconociendo que cada cooperativa se organiza para cumplir unos propósitos específicos, que quien ingresa ha de estar dispuesto a asumir (ser consumidor o usuario en cooperativas que proveen artículos de uso personal, familiar, vivienda o de crédito; ser productor, en cooperativas de colocación de la producción agraria o artesanal o ser obreros o técnicos en las cooperativas de trabajo). Al mismo tiempo, esta afirmación manifiesta de forma implícita que, en ocasiones (cooperativas de trabajo asociado, de viviendas...), la cooperativa sólo puede servir de forma efectiva a un determinado tipo de asociados/as o a un determinado número de ellos, por lo 
que puede haber razones de incapacidad funcional física o de viabilidad por las que una cooperativa pueda imponer un límite al número de asociados/as.

- «Dispuestos a aceptar las responsabilidades..." recuerda a los asociados/as que el ingreso en una entidad de este tipo supone la asunción de una serie de obligaciones, que pueden variar de una cooperativa a otra, pero siempre incluyen la participación en las asambleas, la utilización de los servicios de la cooperativa y el aporte de capital cuando fuere necesario. Es decir, los/las/ aspirantes a socios/as han de estar dispuestos a comprometerse en los flujos de información-decisión, de producción y de distribución y en los flujos financieros.

3. Por último, este principio excluye cualquier tipo de discriminación: «sin discriminaciones raciales, políticas, religiosas, sociales y de género", asumiendo un compromiso general básico de reconocer la dignidad fundamental de todos los individuos, sin discriminaciones de ningún tipo. Concretamente se hace referencia a:

- «la raza». En un principio se consideró que la raza en ningún caso debía aceptarse como criterio para categorizar a los seres humanos, por lo que se planteó incluso omitir su mención, pero tras varios debates entre cooperativistas y expertos del mundo entero se entendió que esa omisión podría acarrear consecuencias erróneas.

— «la política». El movimiento cooperativo siempre ha promovido el trabajo conjunto de personas de diferentes corrientes e ideologías políticas, superando las ideologías tradicionales que tantas tensiones están creando en los últimos tiempos. Es precisamente esta capacidad de reunir personas diferentes en busca de objetivos comunes uno de los grandes alicientes que el movimiento ofrece al siglo XXI.

- «la religión». Las cooperativas deben admitir asociados y asociadas independientemente de sus creencias religiosas. Existen algunas, en su mayoría cooperativas financieras, que son organizadas por comunidades religiosas (Coophalal, cooperativa islámica de servicios financieros ${ }^{4}$ ). Este tipo de organizaciones no invalidan el principio, siem-

4 Se trata de la primera cooperativa de crédito basada en los principios y ética islámicos, que opera inicialmente en Cataluña. 
pre y cuando: a) no impidan la organización de cooperativas similares entre otros grupos religiosos; b) cooperen con ellas; y c) acepten sus responsabilidades de promover el desarrollo general del movimiento cooperativo en sus áreas de influencia.

— «discriminaciones sociales». El término «social» se refiere, sobre todo, a la discriminación basada en clases. Desde sus inicios el movimiento cooperativo se ha esforzado también en reunir a gente de status o clases diferentes. Pero también se refiere a la cultura. Este es un concepto difícil, ya que algunas cooperativas están específicamente organizadas entre grupos culturales (Coopar, cooperativa de las artes representativas; Taller de cantautores coop. ${ }^{5}$ ) Al hilo del argumento anterior, también tienen pleno derecho de existir en tanto: a) no impidan la organización de cooperativas similares entre otros grupos culturales; y b) favorezcan el desarrollo del movimiento cooperativo en sus respectivas áreas.

— «el género». Las cooperativas deben hacer todo lo posible para asegurar que no existan barreras por motivos de género para el ingreso de asociados y asociadas. En ese sentido, una encuesta realizada por la Organización Internacional del Trabajo y la ACl en 2015 puso en evidencia no sólo la extraordinaria eficacia del modelo de cooperativas para ofrecer a las mujeres una manera digna de salir de la pobreza y, con frecuencia, de escapar a la violencia y al abuso, sino también el creciente número de mujeres en cargos directivos, sobre todo en cooperativas de finanzas y seguros. El $80 \%$ de los encuestados opinaban que las cooperativas contribuyen más a la igualdad de género que otro tipo de empresas del sector público o privado. ${ }^{6}$

5 Cooperativas culturales, https://mapacultural.wordpress.com/2009/02/02/cooperativas-culturales/, última consulta 30-05-2017.

6 «Un estudio mundial muestra la creciente participación de las mujeres en las cooperativas», noticia de 9 de marzo de 2015. http://www.ilo.org/global/about-the-ilo/ newsroom/news/WCMS_349688/lang-es/index.htm, última consulta 30-05-2017. Los resultados se trataron poco después en una mesa redonda, "Cooperativas: Igualdad de género y empoderamiento de las mujeres», que tuvo lugar el 10 de marzo en las Naciones Unidas en Nueva York, en un foro organizado como evento paralelo de la 59.a reunión la Comisión de la Condición Jurídica y Social de la Mujer que se llevó a cabo entre el 9 y el 20 de marzo del mismo año. 


\section{II.2. Regulación normativa}

En este apartado nos referiremos a la plasmación concreta de ese principio en los diferentes textos legales que son de aplicación en nuestro ámbito de actuación, haciendo referencia fundamentalmente tanto a la Ley 27/1999, de 16 de julio, de Cooperativas (en adelante LC), como a nuestra ley autonómica, la Ley 4/1993, de 24 de junio, de Cooperativas de Euskadi (en adelante LCE).

Tampoco haremos un análisis exhaustivo del panorama comparado estatal, que podría ser objeto de un trabajo de investigación específico, pero sí mencionaremos algunas diferencias que nos han parecido importantes.

La Ley 27/1999 y la 4/1993 establecen en su artículo $12^{7}$ y 198, respectivamente, los requisitos para ser socio/a de una cooperativa, con un contenido semejante, otorgando gran libertad al respecto:

Artículo 12. Personas que pueden ser socios.

1. En las cooperativas pueden ser socios, en función de la actividad cooperativizada, tanto las personas físicas como jurídicas, públicas o privadas y las comunidades de bienes.

2. Los Estatutos establecerán los requisitos necesarios para la adquisición de la condición de socio, de acuerdo con lo establecido en la presente Ley.

Artículo 19. Personas que pueden ser socios.

1. Pueden ser socios de las cooperativas de primer grado tanto las personas físicas como las jurídicas, públicas o privadas, con las salvedades establecidas en el título II de la presente ley.

En las cooperativas de segundo o ulterior grado se estará a lo dispuesto en el artículo 128.1.

En el momento de la constitución, las cooperativas de primer grado habrán de estar integradas, al menos, por tres socios de la clase de la cooperativa que se constituye. Las de segundo o ulterior grado deberán contar entre sus socios fundadores con dos sociedades cooperativas como mínimo.

2. Podrán adquirir la condición de socios, que se denominarán colaboradores, aquellas personas físicas o jurídicas, públicas o privadas, que, sin poder realizar plenamente el objeto social cooperativo, puedan colaborar en la consecución del mismo. Sus derechos y obligaciones se regularán por lo dispuesto en los Estatutos sociales, y, en lo

7 Ley 27/1999, de 16 de julio, de Cooperativas (https://www.boe.es/buscar/act. php?id=BOE-A-1999-15681, última consulta: 30-05-2017).

8 Ley 4/1993, de 24 de junio, de Cooperativas de Euskadi (https://www.boe.es/buscar/pdf/2012/BOE-A-2012-2011-consolidado.pdf, última consulta: 30-05-2017). 
no previsto por éstos, por lo pactado entre las partes. El conjunto de estos socios, salvo que sean sociedades cooperativas, no podrá ser titular de más de un tercio de los votos, ni en la Asamblea General ni en el Consejo Rector.

3. Los entes públicos con personalidad jurídica podrán ser socios cuando el objeto de la cooperativa sea prestar servicios o realizar actividades relacionadas con las encomendadas a dichos entes, siempre que tales prestaciones no supongan ni requieran el ejercicio de autoridad pública.

A continuación, los artículos $\mathbf{1 3}$ y $\mathbf{2 0}$ se refieren al procedimiento de admisión, con notable semejanza, también en este punto y sólo diferencias de plazos, que concretaremos al hacer referencia al desarrollo estatutario de ambos preceptos. En suma se debe formular una solicitud por escrito al Consejo Rector, que debe resolver en 60 o 90 días, entendiéndose estimada en caso de silencio. Tanto la denegación como la admisión pueden ser recurridas posteriormente ante el Comité de Recursos o, en su defecto, ante la Asamblea General.

\section{Artículo 13. Admisión de nuevos socios.}

1. La solicitud para la adquisición de la condición de socio se formulará por escrito al Consejo Rector, que deberá resolver y comunicar su decisión en el plazo no superior a tres meses, a contar desde el recibo de aquélla, y dando publicidad del acuerdo en la forma que estatutariamente se establezca. El acuerdo del Consejo Rector será motivado. Transcurrido el plazo sin haberse adoptado la decisión, se entenderá estimada.

2. Denegada la admisión, el solicitante podrá recurrir, en el plazo de veinte días, computados desde la fecha de notificación del acuerdo del Consejo Rector, ante el Comité de Recursos o, en su defecto, ante la Asamblea General. El Comité de Recursos resolverá en un plazo máximo de dos meses, contados desde la presentación de la impugnación y la Asamblea General en la primera reunión que se celebre, siendo preceptiva, en ambos supuestos, la audiencia del interesado.

La adquisición de la condición de socio quedará en suspenso hasta que haya transcurrido el plazo para recurrir la admisión o, si ésta fuese recurrida, hasta que resuelva el Comité de Recursos o, en su caso, la Asamblea General.

3. El acuerdo de admisión podrá ser impugnado por el número de socios y en la forma que estatutariamente se determine, siendo preceptiva la audiencia del interesado.

4. En las sociedades cooperativas de primer grado, que no sean de trabajo asociado o de explotación comunitaria de la tierra y en las de segundo grado, los Estatutos podrán prever la admisión de socios 
de trabajo, personas fisicas, cuya actividad cooperativizada consistirá en la prestación de su trabajo personal en la cooperativa.

Serán de aplicación a los socios de trabajo las normas establecidas en esta Ley para los socios trabajadores de las cooperativas de trabajo asociado, con las salvedades establecidas en este artículo.

Los Estatutos de las cooperativas que prevean la admisión de socios de trabajo, deberán fijar los criterios que aseguren la equitativa y ponderada participación de estos socios en las obligaciones y derechos de naturaleza social y económica.

En todo caso, las pérdidas determinadas en función de la actividad cooperativizada de prestación de trabajo, desarrollada por los socios de trabajo, se imputarán al fondo de reserva y, en su defecto, a los socios usuarios, en la cuantía necesaria para garantizar a los socios de trabajo una compensación mínima igual al setenta por ciento de las retribuciones satisfechas en la zona por igual trabajo y, en todo caso, no inferior al importe del salario mínimo interprofesional.

Si los Estatutos prevén un período de prueba para los socios de trabajo, éste no procederá si el nuevo socio llevase al menos en la cooperativa como trabajador por cuenta ajena, el tiempo que corresponde al período de prueba.

5. Para adquirir la condición de socio, será necesario suscribir la aportación obligatoria al capital social que le corresponda, efectuar su desembolso y abonar, en su caso, la cuota de ingreso de acuerdo con lo establecido en los artículos 46 y 52 de esta Ley.

6. Si lo prevén los Estatutos y se acuerda en el momento de la admisión, podrán establecerse vínculos sociales de duración determinada, siempre que el conjunto de estos socios no sea superior a la quinta parte de los socios de carácter indefinido de la clase de que se trate.

La aportación obligatoria al capital social exigible a este tipo de socios no podrá superar el diez por ciento de la exigida a los socios de carácter indefinido y le será reintegrada en el momento en el que cause baja, una vez transcurrido el período de vinculación.

\section{Artículo 20. Admisión.}

1. Los Estatutos establecerán los requisitos necesarios para la adquisición de la condición de socio de acuerdo con lo establecido en la presente ley.

2. La aceptación o la denegación de la admisión no podrán producirse por causas que supongan una discriminación arbitraria o ilicita, en relación con el objeto social.

3. La solicitud de admisión se formulará por escrito a los administradores, que resolverán en un plazo no superior a sesenta días a contar desde la recepción de aquélla, debiendo ser motivada la decisión desfavorable a la admisión. Transcurrido dicho plazo sin resolución expresa se entenderá aprobada la admisión. 
4. Denegada la admisión, el solicitante podrá recurrir ante el Comité de Recursos o, en su defecto, ante la Asamblea General, en el plazo de veinte días desde la notificación de la decisión denegatoria. El recurso deberá ser resuelto por el Comité de Recursos en el plazo de treinta días o, en su caso, por la primera Asamblea General que se celebre, mediante votación secreta. En ambos supuestos será preceptiva la audiencia previa del interesado.

5. El acuerdo de admisión podrá ser recurrido ante el Comité de Recursos o, en su defecto, ante la primera Asamblea General que se celebre, a instancia del número de socios que fijen los Estatutos, que deberán establecer el plazo para recurrir, el cual no podrá ser superior a veinte días desde la notificación del acuerdo de admisión.

La adquisición de la condición de socio quedará en suspenso hasta que haya transcurrido el plazo para recurrir la admisión o, si ésta fuese recurrida, hasta que resuelva el Comité de Recursos o, en su caso la Asamblea General. El Comité de Recursos deberá resolver en el plazo de treinta días y la Asamblea General en la primera reunión que celebre, mediante votación secreta. En ambos supuestos será preceptiva la audiencia previa del interesado.

\section{II.3. Mención estatutaria}

Una vez configurado el marco legal en el que nos desenvolvemos, reviste especial trascendencia su aplicación práctica, es decir, la regulación estatutaria de la admisión de nuevos/as socios/as en cada cooperativa y sus límites. La legislación cooperativa incluye entre los extremos que deben constar en los estatutos sociales, como contenido mínimo y necesario, en primer lugar, la determinación de los requisitos para ser socio/a, dentro del amplio margen concedido por los artículos 12 y 19 de las mencionadas leyes. Sin embargo, la libertad en este punto no es absoluta:

- Límites derivados de los principios configuradores de la cooperativa: puertas abiertas.

- No se puede exigir el cumplimento de requisitos excesivamente difíciles o gravosos que, de facto, configuren una sociedad cerrada (como la realización de aportaciones obligatorias al capital tan elevadas que resulten imposibles de realizar).

- Límites derivados de la Ley en sentido amplio: Constitución y resto del ordenamiento jurídico, con especial mención al principio de igualdad. No pueden incluirse cláusulas atentatorias a los derechos fundamentales. Los requisitos deben poseer carácter objetivo y justificarse en relación al objeto social. En ese sentido 
puede exigirse cierta cualificación profesional, o la posesión de ciertos bienes (titular de explotación agraria en cooperativa agraria) o vincular la condición de socio/a a un cierto ámbito geográfico en el que opera la cooperativa.

- Principio de tipicidad. La solicitud de admisión únicamente puede ser denegada por las causas previstas en la Ley y los Estatutos sociales.

- En cualquier caso, los requisitos que establezcan los Estatutos sociales constituyen una excepción al principio de puertas abiertas y, por tanto, deben ser interpretados restrictivamente.

Una vez delimitadas las condiciones para ser socio/a de la cooperativa, los estatutos han de prever también el procedimiento para su ingreso en la cooperativa.

El interesado ha de presentar por escrito su solicitud al Consejo Rector manifestando su expresa voluntad de pertenecer a la cooperativa. El Consejo Rector debe resolver y comunicar su decisión en un plazo máximo de 60 (LCE) o 90 días (LC). Las leyes extremeña, aragonesa y madrileña, señalan un plazo inferior para que el órgano de administración se pronuncie: 30 días $^{9}$ en el caso de las dos primeras o $45^{10}$, según la Ley 4/1999, de 30 de marzo de Cooperativas de la Comunidad de Madrid. En cualquier caso el Consejo Rector ha de responder mediante escrito motivado (tanto en caso de denegación como de admisión, a modo de garantía frente a cualquier arbitrariedad de los administradores) y ha de atenerse al principio de tipicidad ya mencionado, por lo que sólo podrá denegarse por las causas previstas en la Ley o en los Estatutos sociales. En este punto, algunas leyes autonómicas hilan más fino, precisando las causas que pueden justificar la denegación, además de las dispuestas en los estatutos sociales o en disposiciones normativas: «imposibilidad técnica derivada de las condiciones económico-financieras, organizativas o tecnológicas de la entidad»11;

9 Art. 21 Ley 2/1998, de 26 de marzo, de Sociedades Cooperativas de Extremadura (https://www.boe.es/diario_boe/txt.php?id=BOE-A-1998-12457, última consulta 30-052017) y 17.2 Decreto Legislativo 2/2014, de 29 de agosto, del Gobierno de Aragón, por el que se aprueba el Texto Refundido de la Ley de Cooperativas de Aragón (http://www. boa.aragon.es/cgi-bin/EBOA/BRSCGI?CMD=VEROBJ\&MLKOB=810661044040, última consulta 30-05-2017).

10 Art. 19 Ley 4/1999, de 30 de marzo de Cooperativas de la Comunidad de Madrid (https://www.boe.es/diario_boe/txt.php?id=BOE-A-1999-12334, última consulta: 30-05-2017).

11 Art. 18.4 Ley 14/2011, de 23 de diciembre, de Sociedades Cooperativas Andaluzas (https://www.boe.es/buscar/pdf/2012/BOE-A-2012-877-consolidado.pdf, última consulta: 30-05-2017). 
«imposibilidad técnica, accidental o estructural, debidamente acreditada, derivada de las condiciones económico-financieras, organizativas o tecnológicas ${ }^{12}$; «imposibilidad técnica o estructural debidamente acreditada» ${ }^{13}$.

Pero, ¿y si no hubiera respuesta alguna por parte del Consejo Rector? Tanto la LC, como la LCE y la gran mayoría de las leyes autonómicas sobre cooperativas se decantan por la solución más favorable para el aspirante, es decir, por el silencio positivo: si no hubiera respuesta, se entiende estimada la solicitud. Pero no es así en todos los casos, ya que las leyes gallega, aragonesa, cántabra o de Castilla-León, optan por la alternativa contraria, la desestimación de la solicitud en caso de silencio ${ }^{14}$. En estos casos se puede causar un grave perjuicio al solicitante, cuando la ley únicamente prevé el recurso frente a la denegación expresa, refiriéndose al cómputo del plazo desde la notificación o comunicación de la decisión:

\section{Art. 17.4 (Aragón)}

Contra el acuerdo denegatorio cabrá recurso ante el comité de recursos o, en su defecto, ante la asamblea general, en el plazo de un mes a contar desde su notificación.

Art. 19.4. (Castilla-León)

Denegada la admisión el solicitante podrá recurrir en el plazo de quince días hábiles a contar desde la fecha de notificación del acuerdo denegatorio ante el Comité de Recursos si lo hubiere o ante la primera Asamblea General que se celebre.

Art. 18.3. (Cantabria)

Si se denegase la admisión, el solicitante podrá recurrir, en el plazo de quince días, a contar desde la comunicación de la decisión, ante el comité de recursos, si lo hubiere o ante la primera asamblea general que se celebre.).

12 Art. 29. 4 de la Ley 12/2015, de 9 de julio, de Cooperativas (Cataluña) (https:// www.boe.es/boe/dias/2015/08/14/pdfs/BOE-A-2015-9140.pdf, última consulta: 30-052017).

13 Art. 26.1 Ley 11/2010, de 4 de noviembre, de Cooperativas de Castilla-La Mancha (https://www.boe.es/buscar/pdf/2011/BOE-A-2011-2707-consolidado.pdf, última consulta 30-05-2017).

14 Art. 19.2 Ley 5/1998, de 18 de diciembre, de Cooperativas de Galicia (http:// www.boe.es/diario_boe/txt.php?id=BOE-A-1999-6940, última consulta: 30-05-2017); Art. 18.2 Ley 6/2013, de 6 de noviembre, de Cooperativas de Cantabria (https://www. boe.es/diario_boe/txt.php?id=BOE-A-2013-12424, última consulta: 30-05-2017); Art. 19.3 Ley 4/2002, 11 abril, de Cooperativas de la Comunidad de Castilla y León (https:// www.boe.es/diario_boe/txt.php?id=BOE-A-2002-9331, última consulta: 30-05-2017); Art. 17.2 Decreto Legislativo 2/2014 (Aragón). 
Para evitar esa indefensión, entendemos que el aspirante mantiene, en todo caso, su derecho a recurrir frente a la denegación expresa o por silencio, computándose en este último caso el plazo para hacerlo desde la terminación del plazo del Consejo Rector para resolver, como establece expresamente la ley gallega ${ }^{15}$.

Por lo tanto, denegada la admisión (de forma expresa o por silencio), el solicitante podrá recurrir, ante el Comité de Recursos o, en su defecto, ante la Asamblea General, en el plazo de $\mathbf{2 0}$ días. Son muchas las leyes autonómicas que amplían ese plazo a un mes ${ }^{16}$ y sólo tres las que lo reducen a 15 días (Castilla-León, Murcia y Cantabria ${ }^{17}$ ). El recurso deberá ser resuelto por el Comité de Recursos en el plazo máximo de 30 (LCE) o 60 días (LC); sólo la ley catalana establece un plazo superior de 3 meses $^{18}$ o, en su caso, por la primera Asamblea General que se celebre, mediante votación secreta. En ambos supuestos será preceptiva la audiencia previa del interesado.

Y una vez agotada la vía cooperativa ¿cabe el recurso a la vía judicial? Algunas normas (ley andaluza, aragonesa, catalana, madrileña, riojana, o Castilla Mancha) ${ }^{19}$ contemplan expresamente la posibilidad del solicitante de impugnar el acuerdo que resuelve el recurso contra la denegación o la admisión. En cambio otras, como la LC o la LCE guardan silencio al respecto; por tanto, se plantea la duda de si existe la posibilidad de que el solicitante pueda recurrir dicho acuerdo ante los órganos judiciales competentes. Sobre este extremo, la doctrina se

15 Art. 19.2 Ley 5/1998 (Galicia).

16 Art. 23.3 Ley 1/2003, de 20 de marzo, de Cooperativas de las Illes Balears (https://www.boe.es/diario_boe/txt.php?id=BOE-A-2003-7872, última consulta: 30-05-2017); Art. 21.3 Ley 2/1998 (Extremadura); Art. 19.2 Ley 5/1998 (Galicia); Art. 19.3 Ley 4/1999 (Madrid); Art. 29.5 Ley 12/2015 (Cataluña); Art. 21.3 Ley 4/2001, de 2 de julio, de Cooperativas de la Rioja (https://www.boe.es/buscar/doc. php?id=BOE-A-2001-13944, última consulta 30-05-2017); Art. 17.5 Decreto Legislativo 2/2014 (Aragón); Art. 20. 2 Decreto Legislativo 2/2015, de 15 de mayo, del Consell, por el que aprueba el texto refundido de la Ley de Cooperativas de la Comunitat Valenciana (https://www.boe.es/buscar/pdf/2015/DOCV-r-2015-90416-consolidado. pdf, última consulta: 30-05-2017); Art. 22.2 Ley Foral 14/2006, de 11 de diciembre, de Cooperativas de Navarra. (http://www. lexnavarra.navarra.es/detalle.asp?r=5420, última consulta: 30-05-2017).

17 Art. 19.4, Ley 4/2002 (Castilla y León); Art. 23.2 Ley 8/2006, de 16 de noviembre, de Sociedades Cooperativas, de la Región de Murcia (https://www.boe.es/diario_ boe/txt.php?id=BOE-A-2007-9417, última consulta: 30-05-2017); Art. 18.3 Ley 6/2013 (Cantabria).

18 Art. 29.5 Ley $12 / 2015$.

19 Art. 18.5 Ley 14/2011 (Andalucía); art. 17.4 Decreto Legislativo 2/2014 (Aragón); art. 29.6 Ley 12/2015 (Cataluña); art. 19.6 Ley 4/1999 (Madrid); art. 21.5 Ley 4/2001 (la Rioja); art. 26.4 Ley 11/2010 (Castilla-Mancha) 
ha mostrado dividida, pero coincido con quienes responden afirmativamente, dado que el solicitante es titular, cuando menos, de un interés legítimo, que le otorgaría amparo conforme al artículo 24 de la Constitución, argumento que vemos reforzado por las legislaciones asturiana y valenciana que sí mencionan expresamente el «derecho a la admisión» ${ }^{20}$. Se trata, en cualquier caso, de una conclusión lógica puesto que cualquier limitación o restricción del derecho constitucional a la tutela judicial no se presume y debe estar expresamente prevista en la ley en términos que no ofrezcan posibilidad de dobles interpretaciones, tal y como sucede con el intento de conciliación en el orden social o con el agotamiento previo de la vía administrativa en el orden contencioso-administrativo.

Finalmente, expuestos los requisitos y el procedimiento de admisión, se plantea la duda sobre el alcance del poder de decisión de la cooperativa. En virtud de este principio de adhesión voluntaria y abierta, de su regulación normativa y posterior desarrollo estatutario ¿ha de entenderse que la persona que quiera ingresar en una cooperativa tiene un derecho subjetivo a exigir su alta en la entidad (tal y como menciona expresamente la ley valenciana o asturiana) o, por el contrario, han de admitirse límites a dicho principio? Dicho de otra forma ¿está la cooperativa obligada a admitir a cualquier persona que reúna los requisitos que establezcan los Estatutos sociales, tras una mera verificación formal? o, por el contrario ¿tienen los administradores el derecho y el deber de examinar y valorar si el aspirante debe o no ser admitido?

Entendemos que el punto de partida ha de ser una clara distinción de conceptos: no es lo mismo el cumplimiento de los requisitos para el ingreso (condición esencial para poder aspirar al mismo) y la admisión en sí (acto que requiere de la voluntad expresa o tácita del órgano competente para admitir asociados/as). En ese sentido, consideramos que debe primar el carácter personalista de estas entidades, la importancia de la persona del socio/a, que justifican la posibilidad de dejar a discreción de la cooperativa admitir o no, a una persona que, cumpliendo con todos los requisitos exigidos, manifiesta su voluntad de asociarse. Entendemos que no por ello quiebra el principio de puertas abiertas, que queda suficientemente garantizado mediante la posibilidad de impugnación interna y externa de las decisiones de los órganos cooperativos (Comité de Recursos o Asamblea General).

20 Art. 22. 1. Ley 4/2010, de 29 de junio, de Cooperativas (Asturias) https://www. boe.es/buscar/pdf/2010/BOE-A-2010-14628-consolidado.pdf, última consulta 30-052017; art. 20 Decreto Legislativo 2/2015 (Valencia). 


\section{Las aportaciones}

Sin embargo no termina ahí el periplo del/la aspirante a socio/a para conseguir su objetivo. Para adquirir la condición de socio/a, será necesario suscribir, además, la aportación obligatoria al capital social que le corresponda, efectuar su desembolso y abonar, además, una cuota de ingreso de acuerdo con lo establecido en cada caso. Por lo tanto, nos referiremos también brevemente a este aspecto.

Efectivamente, tras superar el proceso de admisión, el solicitante ha de cumplir con su primera obligación societaria: la realización de la aportación mínima fijada en los estatutos, que podrá ser diferente para las distintas clases de socios/as o para cada socio, en proporción al compromiso o uso potencial que cada uno asuma en la actividad cooperativizada. En ese sentido, los estatutos han de fijar como contenido mínimo la aportación obligatoria mínima al capital social para ser socio/a, la forma y plazos de desembolso y los criterios para fijar la aportación obligatoria que habrán de efectuar los nuevos socios/as que se incorporen a la cooperativa.

Esta aportación ha de desembolsarse al menos en un $25 \%$ en ese momento y el resto en la forma y plazos que determinen los estatutos o en su defecto la Asamblea General.

Normalmente estas aportaciones suelen ser dinerarias, pero también pueden consistir en bienes y derechos, si así lo prevén los estatutos. En éste último caso el Consejo Rector deberá fijar su valoración, previo informe de uno o varios expertos independientes y responderá solidariamente de la realidad de dichas aportaciones y del valor que se les haya atribuido. No obstante, los estatutos pueden establecer que la valoración realizada por el Consejo Rector sea aprobada por la Asamblea General, en cuyo caso se le exime de la mencionada responsabilidad.

Además de la aportación mínima obligatoria, en ocasiones la cooperativa exige también una "cuota de ingreso" a los nuevos socios/as, con el objetivo de evitar la pérdida de valor de las participaciones de los antiguos socios/as, como resultado del aumento del número de éstos que tienen derecho a participar del patrimonio neto. Se pretende, por lo tanto, eliminar la disminución del coeficiente patrimonio neto/socio, que se produce con la entrada de nuevos socios/as sin un aumento proporcional del patrimonio social. Por lo tanto, para soslayar dicha pérdida de valor se exige al nuevo socio/a que aporte una cuota de ingreso de forma que su entrada vaya acompañada de un aumento proporcional del patrimonio neto. 


\section{Conclusión}

A modo de conclusión señalaremos brevemente que las cooperativas realizan un planteamiento más personal que las sociedades anónimas al relacionarse con los socios y socias o con los accionistas, respectivamente, en todos los aspectos, con las consiguientes ventajas e inconvenientes que ello comporta. Como inconveniente podría señalarse la supeditación de la entrada a la voluntad de la cooperativa, manifestada a través de los administradores o de la Asamblea General. Sin embargo, consideramos que esa inicial "desventaja» constituye, a su vez, una clara ventaja, al suponer una mayor garantía de vinculación o compromiso para con la cooperativa del socio/a que ha superado el examen lo que, sin duda, redundará en un mejor funcionamiento de la entidad.

\section{Bibliografía y otras fuentes}

CARRASCO CARRASCO, M. 1991. "La Empresa Cooperativa actual: ni mutualidad ni ausencia del lucro. La justificación de una protección fiscal», en CIRIEC-España, Revista de Economía Pública, Social y Cooperativa, n. ${ }^{\circ} 14$ : $1-18$.

MIRANDA, J. E. 2012. De la crisis de identidad al rescate de la génesis del cooperativismo. Madrid: Dykinson.

MLADENATZ, G. 1969. Historia de las doctrinas cooperativas. Buenos Aires: Intercoop.

MÖLLER, E. 1986. El cooperativismo como proceso de cambio. Doctrina histórica del cooperativismo. Bolivia: Editorial Los Amigos del Libro.

KAPLAN DE DRIMER, A. y DRIMER B. 1981. Las cooperativas. Fundamentos. Historia. Doctrina. Buenos Aires: Intercoop.

URIBE GARZÓN, C. 2001. Bases del cooperativismo. Colombia: Editorial Guadalupe Ltda.

\section{Legislación}

Ley 4/1993, de 24 de junio, de Cooperativas de Euskadi.

Ley $2 / 1998$, de 26 de marzo, de Sociedades Cooperativas de Extremadura.

Ley 5/1998, de 18 de diciembre, de Cooperativas de Galicia.

Ley 4/1999, de 30 de marzo de Cooperativas de la Comunidad de Madrid.

Ley 27/1999, de 16 de julio, de Cooperativas.

Ley 4/2001, de 2 de julio, de Cooperativas de la Rioja.

Ley 4/2002, 11 abril, de Cooperativas de la Comunidad de Castilla y León.

Ley 1/2003, de 20 de marzo, de Cooperativas de las Illes Balears. 
Ley 8/2006, de 16 de noviembre, de Sociedades Cooperativas, de la Región de Murcia.

Ley Foral 14/2006, de 11 de diciembre, de Cooperativas de Navarra.

Ley 4/2010, de 29 de junio, de Cooperativas (Asturias).

Ley 11/2010, de 4 de noviembre, de Cooperativas de Castilla-La Mancha.

Ley 14/2011, de 23 de diciembre, de Sociedades Cooperativas Andaluzas.

Ley 6/2013, de 6 de noviembre, de Cooperativas de Cantabria.

Ley 12/2015, de 9 de julio, de Cooperativas (Cataluña).

Decreto Legislativo 2/2014, de 29 de agosto, del Gobierno de Aragón, por el que se aprueba el Texto Refundido de la Ley de Cooperativas de Aragón.

Decreto Legislativo 2/2015, de 15 de mayo, del Consell, por el que aprueba el texto refundido de la Ley de Cooperativas de la Comunitat Valenciana.

Fuentes en soporte electrónico

"Los estatutos de la cooperativa de Rochdale. Gestión participativa»

http://www.gestionparticipativa.coop/portal/index.php?option=com content\&view=article\&id=254:los-estatutos-de-la-cooperativa-de-rochdale \&catid=37: getaways\&ltemid=364

«Cooperativas culturales»

https://mapacultural.wordpress.com/2009/02/02/cooperativas-culturales/

«Un estudio mundial muestra una creciente participación de las mujeres en las cooperativas»

http://www.ilo.org/global/about-the-ilo/newsroom/news/WCMS_349688/lang-es/index.htm 


\section{Derechos de autor}

El Boletín de la Asociación Internacional de Derecho Cooperativo es una revista de acceso abierto lo que significa que es de libre acceso en su integridad inmediatamente después de la publicación de cada número. Se permite su lectura, la búsqueda, descarga, distribución y reutilización legal en cualquier tipo de soporte sólo para fines no comerciales y según lo previsto por la ley; sin la previa autorización de la Editorial (Universidad de Deusto) o el autor, siempre que la obra original sea debidamente citada (número, año, páginas y DOI si procede) y cualquier cambio en el original esté claramente indicado.

\section{Copyright}

The International Association of Cooperative Law Journal is an Open Access journal which means that it is free for full and immediate access, reading, search, download, distribution, and lawful reuse in any medium only for non-commercial purposes, without prior permission from the Publisher or the author; provided the original work is properly cited and any changes to the original are clearly indicated. 